\title{
Low-molecular-weight heparin and intermittent pneumatic compression for thromboprophylaxis in critical patients
}

\author{
BING WAN ${ }^{1}$, HAI-YAN FU ${ }^{2}$, JIANG-TAO YIN ${ }^{1}$ and GUO-QING REN ${ }^{3}$ \\ ${ }^{1}$ Department of Intensive Care Unit; ${ }^{2}$ Department of General Surgery; ${ }^{3}$ Department of Emergency Medicine, \\ Affiliated Hospital of Jiangsu University, Zhenjiang, Jiangsu 212001, P.R. China
}

Received September 30, 2014; Accepted September 24, 2015

DOI: $10.3892 /$ etm.2015.2795

\begin{abstract}
The efficacy and safety of physiotherapeutic prophylaxis for venous thromboembolism in critically ill patients with heparin contraindication remains unclear. In the present study it was hypothesized that physiotherapy prophylaxis with intermittent pneumatic compression (IPC) would be safe and effective for patients unable to receive low-molecular-weight heparin (LMWH). In addition, this study investigated whether a combined therapy of IPC with LMWH would be more effective for the prophylaxis of deep vein thrombosis (DVT) in critical patients. A total of 500 patients were divided into four groups according to the prophylaxis of DVT. The IPC group consisted of 95 patients with heparin contraindication that received IPC treatment; the LMWH group consisted of 185 patients that received an LMWH injection; the LMWH + IPC group consisted of 75 patients that received IPC treatment and LMWH injection; and the control group consisted of 145 patients that received no IPC treatment or injection of LMWH. Each patient was evaluated clinically for development of DVT and the diagnosis was confirmed by Doppler study. Venous thromboembolism was a common complication among the trauma patients with severe injuries. Patients responded positively to the treatment used in the intervention groups. Patients exhibited an improved response to LMWH + ICP compared with IPC or LMWH alone, while no significant difference was detected between the IPC and LMWH groups. These results were applicable to patients that had a Wells score of $\geq 3$; however, no significant differences in DVT incidence were observed among the patients who had a Wells score of $<3$. In this observational study, LMWH + ICP appeared to be more effective than either treatment alone in treating critically ill trauma patients with severe injuries that are at high risk for VTE and bleeding simultaneously.
\end{abstract}

Correspondence to: Dr Bing Wan, Department of Intensive Care Unit, Affiliated Hospital of Jiangsu University, 438 Jiefang Road, Zhenjiang, Jiangsu 212001, P.R. China

E-mail: andiantong@163.com

Key words: deep venous thrombosis, low-molecular-weight heparin, intermittent pneumatic compression

\section{Introduction}

Pulmonary embolism (PE) and deep vein thrombosis (DVT), referred to collectively as venous thromboembolism (VTE), are life-threatening conditions that may arise in patients following acute trauma and major surgery, particularly after total hip and knee replacement $(1,2)$. Patients are usually asymptomatic until the occurrence of a fatal PE (3). Prevention of VTE requires a reliable tool for the stratification of the risk for developing VTE, screening strategies and effective prophylaxis to significantly reduce mortality in intensive care unit (ICU) patients. Basic indications of VTE, including endothelial abnormality, stasis of blood flow and hypercoagulability, are typically observed in the critically ill (4). Pharmacological prophylaxis with low-molecular-weight heparin (LMWH) has been demonstrated to reduce VTE rates $(5,6)$. In addition, LMWH may be associated with a reduction in major bleeding compared with unfractionated heparin (7). Furthermore, the trade-off between VTE prevention and excess hemorrhage has prevented the development of a consensus in the guidelines of major professional societies (8). The reliable identification of patients that could potentially benefit from high-potency prophylaxis may help to resolve this controversy and facilitate the selection of prophylaxis by ICU teams. Previous studies on the thromboprophylactic effect of intermittent pneumatic compression (IPC) have indicated that its use may protect against major DVT events (9-11). However, compliance has previously been a limitation, and this treatment is not applicable to patients with a pulmonary edema or heart failure. To the best of our knowledge, no study has directly compared the thromboprophylactic effect of IPC with that of LMWH. Therefore, the aim of the present study was to compare the efficacy and safety of IPC combined with LMWH, IPC and LMWH as VTE prophylactic treatments in 500 patients undergoing major treatment in an ICU.

\section{Materials and methods}

Study design and patient enrolment. This retrospective study was performed in the general and surgical ICU of the Affiliated Hospital of Jiangsu University (Zhenjiang, China) between January 2010 and March 2014. After the trial was approved by the Institutional Ethics Committee, 500 patients were enrolled following admission to the ICU. Informed consent was 
obtained either from the patient or the patient's family. The causes of ICU admission were various and included: Traumatic brain injury and cerebral infarction; multiple injuries such as multiple rib fractures, hemothorax with pneumothorax, pulmonary contusion, ruptured spleen and intestinal rupture; orthopedic hip replacement, pelvic fractures and femoral shaft fractures; cancer such as lung, esophageal, stomach, colon and pancreatic cancer, in addition to post-operative treatment following the removal of other tumors; severe acute pancreatitis, cirrhosis decompensation, severe pneumonia and acute respiratory distress syndrome; shock, multiple organ dysfunction syndrome and cardiopulmonary arrest resuscitation, and active peptic ulcer, acute infective endocarditis, hemorrhagic stroke and severe renal impairment (Table I). The patients were divided into four groups according to the prophylaxis of DVT, as follows: IPC group, LMWH group, LMWH + IPC group and control group (in which the patients were not treated with IPC or LMWH) (Fig. 1). Severity of patient illness was evaluated using the Glasgow Coma Scale, Wells' scoring system and an assessment of muscle force.

Treatment. Patients that had been admitted to the ICU for $<30$ days were excluded from the study.

Risk factors of all patients were assessed and scored (12): Low risk, 1 point; medium risk, 2 points; high risk, 3 or 4 points; and ultra-high risk, $\geq 5$ points. Higher scores indicated a higher risk of developing DVT. Patients who had heparin contraindication were grouped into a physiotherapy group and received the IPC intervention (Shengsi Haichuan Medical Equipment, Co., Ltd., Shanghai, China) for $2 \mathrm{~h}$ each time, twice a day; patients in the LMWH and LMWH + IPC groups received LMWH by subcutaneous injection if their platelet count and prothrombin time were within the normal ranges $(5,000$ units by subcutaneous injection, every $12 \mathrm{~h}$ ). The dose was changed on day 3 (5,000 units by subcutaneous injection, once daily) to maintain the prothrombin time at 1.5-2.0-fold the normal value.

The four chambers of the IPC device (Wonjin manufacturer, Shengsi Haichuan Medical Equipment Co. Ltd., Shanghai, China) were placed on the calf, with two placed on the thigh (avoiding the knee). Then, pneumatic pressure was adjusted from the ankle to the calf and thigh, so that the pressure on the ankle was the highest, and the pressure on the thighs was the lowest, in order to promote effective venous return from the limb. Pressure was increased rapidly to evacuate the deep vein of the lower extremity within $11 \mathrm{sec}$, and subsequently reduced for $60 \mathrm{sec}$. Finally, complete refilling of the deep vein was allowed.

The entire leg from the ankle to the thigh was wrapped in inflatable sleeves for IPC, allowing for an appropriate space of $\leq 1 \mathrm{~cm}$ between the IPC sleeve and the limb. The limb skin temperature, color and dorsalis pedis artery pulse were monitored closely by a nurse using an electronic thermometer and by manual measurement.

Evaluation criteria. Simplified Wells' scoring systems were used to evaluate DVT and PE (13). The most common symptoms of DVT include: Pain deep in the calf or thigh, unilateral swelling, increased temperature of the leg, tenderness and redness. Positive signs for DVT additionally included tenderness of the deep vein, swelling of the lower limb or calf circumference $>3 \mathrm{~cm}$ more than the normal size $(10 \mathrm{~cm}$ below tibial tuberosity), limb or pitting edema of the lower extremities and visible superficial veins (excluding varicose veins) (14-16). Heparin contraindications include: i) Allergic reaction to heparin and LMWH; ii) severe coagulopathy; iii) history of reduced platelet count induced by treatment with LMWH or heparin; iv) active peptic ulcer or bleeding tendency of organ damage; and v) acute infective endocarditis, with the exception of infection following heart valve replacement surgery.

Color Doppler ultrasonography. Over the last two decades, venous compression ultrasonography has become the imaging test of choice for diagnosing DVT in the lower extremities of patients. Ultrasonography for detection on venous thromboembolism has been used widely (17-19).

Ultrasound examination (LOGIQ P3 ultrasound system; GE Healthcare, Shanghai, China) of the symptomatic leg(s) was performed by compression of the proximal veins. Compression maneuvers of the symptomatic leg were conducted at $1-\mathrm{cm}$ intervals along the length of the femoral vein (from the inguinal ligament) and popliteal veins to the level of the calf vein trifurcation (20).

In addition, the ipsilateral external iliac vein was imaged in all patients. Intraluminal echogenic masses consistent with a thrombus were noted on B-mode imaging, and spontaneous venous flow was assessed using Doppler interrogation. Absent or reduced flow was further evaluated via ultrasound apparatus (20). DVT was diagnosed based on noncompressibility at any two contiguous segments of the femoral or popliteal vein. Furthermore, DVT was diagnosed in the iliac veins by the absence of flow within the iliac vein and/or the presence of a visible thrombus by Doppler imaging. DVT was excluded based on full compressibility of the femoral and popliteal veins and normal Doppler imaging of the iliac veins.

Imaging was performed by trained technicians, and the images were reviewed by local radiologists. In all cases, abnormal results were confirmed by the local radiologist.

Ultrasound measurements for the deep vein of a lower extremity were performed on days 1, 3,7 and 14 after admission. Ultrasound diagnostic criteria for lower extremity DVT (21) included: Widening of deep vein lumen of the affected region after thrombosis, observation of substantial hypoechoic lumen, filled or occupied portions of affected deep vein, force required to compress the affected region, inability to flatten the venous lumen and irregularly shaped thrombus. The affected region (thrombus) showed no color flow signals on the screen of the B-mode ultrasonic diagnostic equipment (GE Healthcare) when the venous lumen was completely blocked. When the lumen was partially blocked, reduced blood flow with an outline containing points or in a scattered pattern could be observed near the middle or edge of the thrombus. In certain cases blood flow appeared as spots when the distal limb was compressed.

Enhanced helical computed tomography (CT) examination (SOMATOM Definition AS 64-slice spiral CT scanner; Siemens AG, Munich, Germany) was used in patients with symptoms highly suggestive of DVT but with negative findings on ultrasonography. 
Table I. Comparison of basic characteristics among the four patient groups.

\begin{tabular}{|c|c|c|c|c|}
\hline Characteristic & IPC & LMWH & LMWH + ICP & Control \\
\hline Total cases & 95 & 185 & 75 & 145 \\
\hline Gender (male/female) & $65 / 30$ & $125 / 60$ & $55 / 20$ & $110 / 35$ \\
\hline Age (mean years $\pm \mathrm{SE})$ & $55.0 \pm 4.0$ & $58.9 \pm 3.4$ & $59.6 \pm 3.5$ & $60.3 \pm 3.3$ \\
\hline Craniocerebral trauma & 9 & 18 & 7 & 11 \\
\hline Brain infarction & 5 & 36 & 10 & 12 \\
\hline Multiple injury & 6 & 14 & 3 & 12 \\
\hline Tumor postoperative & 3 & 22 & 10 & 11 \\
\hline Severe acute pancreatitis & 5 & 28 & 9 & 12 \\
\hline Decompensation of cirrhosis & 4 & 12 & 10 & 8 \\
\hline Severe pneumonia & 1 & 18 & 6 & 8 \\
\hline Acute respiratory distress syndrome & 3 & 14 & 5 & 6 \\
\hline Hemorrhagic shock & 12 & 1 & 2 & 15 \\
\hline Multiple organ dysfunction syndrome & 3 & 9 & 5 & 12 \\
\hline Cardiopulmonary resuscitation & 4 & 12 & 7 & 5 \\
\hline Active peptic ulcer & 9 & 0 & 0 & 4 \\
\hline Acute infective endocarditis & 8 & 0 & 0 & 6 \\
\hline Hemorrhagic stroke & 14 & 0 & 0 & 14 \\
\hline Severe renal impairment & 9 & 1 & 1 & 9 \\
\hline
\end{tabular}

IPC, intermittent pneumatic compression; LMWH, low-molecular-weight heparin; SD, standard deviation.

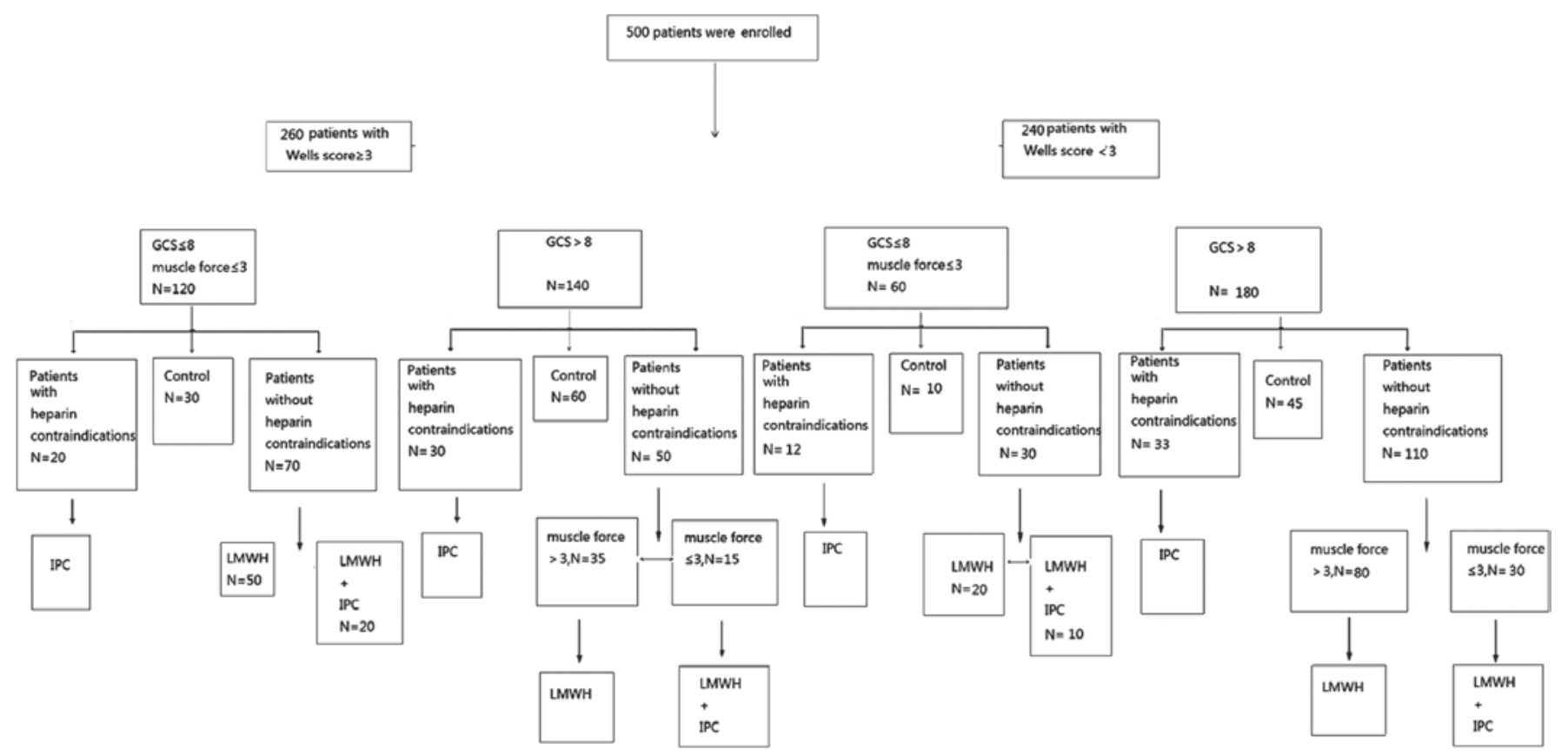

Figure 1. Patient enrollment, grouping and treatment. GCS, Glasgow Coma Scale; IPC, intermittent pneumatic compression; LMWH, low-molecular-weight heparin.

Statistical analysis. Ages are presented as the mean \pm standard error, and gender, rates of DVT and PE are presented as percentages. Statistical analysis was conducted using SPSS software, version 13.0 (SPSS, Inc., Chicago, IL, USA). Rates among the four groups were compared using $\chi^{2}$ tests, whereas means among the four groups were compared using t-tests. Ages among the four groups were compared using analysis of variance. $\mathrm{P}<0.05$ was considered to indicate a statistically significant difference.

\section{Results}

Incidence of DVT. A total of 500 patients were divided into four groups: IPC, LMWH, LMWH + ICP or control (without 
Table II. Incidence of DVT, PE and complications of treatment in the four groups.

\begin{tabular}{lcccc}
\hline Group & No. of patients & DVT cases, $\mathrm{n}(\%)$ & PE cases, $\mathrm{n}(\%)$ & Bleeding cases, $\mathrm{n}(\%)$ \\
\hline IPC & 95 & $9(9.5)^{\mathrm{a}, \mathrm{b}}$ & $3(3.2)^{\mathrm{a}}$ & 0 \\
LMWH & 185 & $17(9.2)^{\mathrm{a}, \mathrm{b}}$ & $2(1.1)^{\mathrm{a}}$ & $10(5.4)$ \\
LMWH + IPC & 75 & $0(0)^{\mathrm{a}}$ & $0(0)^{\mathrm{a}}$ & $4(5.3)$ \\
Control & 145 & $34(23.4)$ & $20(13.6)$ & 0 \\
\hline
\end{tabular}

${ }^{\mathrm{a}} \mathrm{P}<0.01$ vs. the control group; ${ }^{\mathrm{b}} \mathrm{P}<0.01$ vs. the LMWH + IPC group. DVT, deep vein thrombosis; PE, pulmonary embolism; IPC, intermittent pneumatic compression; LMWH, low-molecular-weight heparin.

Table III. Incidence of DVT in patients from the four groups with a Wells score $\geq 3$.

\begin{tabular}{lcc}
\hline Group & No. of patients & DVT cases, $\mathrm{n}(\%)$ \\
\hline IPC & 50 & $7(14)^{\mathrm{a}, \mathrm{b}}$ \\
LMWH & 85 & $15(17.6)^{\mathrm{a}, \mathrm{b}}$ \\
LMWH + IPC & 35 & $0(0)^{\mathrm{a}}$ \\
Control & 90 & $32(35.4)$ \\
\hline
\end{tabular}

${ }^{\mathrm{a}} \mathrm{P}<0.01$ vs. control group; ${ }^{\mathrm{P}}<0.01$ vs. LMWH + IPC group. DVT, deep vein thrombosis; IPC, intermittent pneumatic compression; LMWH, low-molecular-weight heparin.

LMWH or IPC). The incidence rates of DVT were $9.5 \%$ in the IPC group, $9.2 \%$ in the LMWH group, $0 \%$ in the LMWH + ICP group and $23.4 \%$ in the control group. Statistically significant significances were detected between the three prophylaxis groups and the control group, and were also noted between the LMWH + ICP group and the IPC and LMWH groups. No statistically significant difference was observed in DVT incidence between the IPC group and the LMWH group (Table II).

Incidence of PE. The incidence rates of PE were $3.2 \%$ in the IPC group, $1.1 \%$ in the LMWH group, $0 \%$ in the LMWH + ICP group and $13.6 \%$ in the control group. There were observed to be statistically significant differences between the three prophylaxis groups and the control group, but no statistically significant differences were noted in PE incidence among the prophylaxis groups (Table II).

Incidence of hemorrhage. The incidence rates of hemorrhage complications were $0 \%$ in the IPC group, $5.4 \%$ in the LMWH group, $5.3 \%$ in the LMWH + IPC group and $0 \%$ in the control group. Patients that presented with subcutaneous hemorrhage complications in the LMWH and LMWH + IPC groups stopped bleeding following drug withdrawal, and no patients suffered hemorrhage complications in the IPC and control groups.

Wells' scoring system. Among the patients that had a Wells score of $\geq 3$, the incidence of DVT was $14 \%$ in the IPC group, $17.6 \%$ in the LMWH group, $0 \%$ in the LMWH + IPC group and $35.4 \%$ in the control group. Results showed statistically significant differences between the three prophylaxis groups and the control group and between the LMWH + IPC group and the IPC and LMWH groups. No statistically significant differences were identified between the IPC and LMWH groups (Table III). Among the patients with a Wells score of $<3$ (data not shown), the incidences of DVT were $4.4 \%$ in the IPC group, $2 \%$ in the LMWH group, $0 \%$ in the LMWH + IPC group and $3.6 \%$ in the control group. These results showed no statistically significant differences among the four groups.

\section{Discussion}

Prophylaxis of VTE in critically ill patients that are at a high risk for thrombosis and bleeding simultaneously poses a major challenge (22), as in a previous study it was found that $22 \%$ of trauma patients who had ongoing bleeding or injuries were at high risk for serious bleeding complications (23). The observation that the risk of VTE increases as the number of risk factors increases should be taken into account when assessing risk levels and considering thromboprophylaxis (24). The American College of Chest Physicians and the American Society of Colon and Rectal Surgeons have recommended that every hospital should formulate a risk stratification approach to categorize trauma patients in terms of thromboembolic risk (25). With improvements in detection methods, DVT and PE detection rates have increased; however, the methods for preventing DVT require further improvement. Due to its simplicity of use, IPC has been used in our department for several years, despite reports that further research is required to observe the validity of IPC device for preventing VTE (26).

As patients may be admitted to a general ICU for a wide variety of reasons, a single method for preventing DVT may have limitations. In clinical practice, patients that have suffered trauma or fracture of the leg are not good candidates for the use of IPC, whereas in other cases, such as patients suffering hypovolemic shock, trauma or other coagulation disorders, there is a risk of bleeding with heparin use. However, LMWH is associated with a low risk of bleeding (27). Numerous reports have indicated that LMWH is able to reduce the incidence of DVT in patients with cancer and patients undergoing major surgery (28-30), in a dose-dependent manner (31). Dennis et al (32) reported that IPC was effective for DVT prophylaxis. Sekine and Koh (33) recommended IPC for DVT prophylaxis in patients with low or medium risk and low dose heparin for high-risk patients. 
IPC is an effective mechanical method of DVT prophylaxis, exerting an antithrombotic effect that appears to be a result of reduced plasminogen activator inhibitor-1 levels and increased tissue plasminogen activator activity. These actions stimulate fibrinolytic activity and increase venous blood flow velocity, thereby reducing stasis and altering hypercoagulability (34). However, the efficacy of this treatment is determined in part by patient compliance with the treatment protocol. Furthermore, the use of ICP is limited in cases with certain diseases such as pulmonary edema and non-congestive heart failure.

LMWH is among the most significant recent developments in prophylaxis against DVT (24). The anticoagulatory effects of LMWH have been well characterized and function by reducing the activity of coagulation factor XIIa and thrombin (35). LMWH interacts with platelets and has been reported to inhibit platelet aggregation, thus prolonging the duration of bleeding in patients. Thus, the clinical application of LMWH remains limited by its primary side-effect, bleeding (35). Although it is likely that the hemorrhaging observed in the present study was associated with the anticoagulatory effects of LMWH, other factors may also be important. The evaluation of efficacy was based on the frequency of VTE (36). It was observed that there was a statistical significance between the three prophylaxis groups and the control group, which indicated the efficacy of those treatments. ICP may exert its antithrombotic effect by stimulating fibrinolytic activity and increasing venous blood flow velocity, while LMWH is well characterized and functions by accelerating the inhibitory effect of antithrombin on factors XIIa, XIa, Xa and IXa and thrombin (24), whether the IPC and LMWH treatments produced a significant difference in efficacy was of no importance. However, by combining ICP and LMWH treatments, an improved response may be achieved as each treatment functions via an independent mechanism.

The results of the present study require interpretation within the context of its limitations. Firstly, this study did not involve true randomization, and was an observational study since the allocation of treatment was determined by the provider not by a random sequence. However, this protocol was necessary as certain patients exhibited heparin contraindications or pulmonary edema and were unable to receive certain treatments. Secondly, it remains unclear whether an improved benefit/risk ratio of prophylaxis of thromboembolic events versus risk of bleeding complications may be established for LMWH (24). LMWH-induced thrombocytopenia has been reported in clinical therapy (37). In addition, it was observed in the present study that the LMWH + ICP treatment had a significant improvement in prophylactic effect, with decreased side effects, which provided a better prospective for the prevention of thromboprophylaxis in critically ill patients.

In conclusion, the results of the present study suggest that LMWH combined with IPC exhibited an excellent prophylactic effect against DVT and PE. The effect of IPC was comparable to that of LMWH anticoagulation therapy. As for the patients in the ICU who were at high risk for DVT but had contraindications for heparin, this therapy may effectively reduce the incidence of DVT if administered selectively on the basis of the patient's condition. For patients at a high risk of DVT, with no contraindications for heparin, it is proposed that
IPC in combination with anticoagulant therapy may effectively reduce the incidence of DVT and is potentially applicable in a clinical context.

\section{Acknowledgements}

This study was supported by Zhenjiang City Technology Support Program-Social Development Project (no. SH2013037) and Clinical Science and Technology Development Fund of Jiangsu University (no. JLY20120164).

\section{References}

1. Geerts WH, Jay RM, Code KI, Chen E, Szalai JP, Saibil EA and Hamilton PA: A comparison of low-dose heparin with low-molecular-weight heparin as prophylaxis against venous thromboembolism after major trauma. N Engl J Med 335: 701-707, 1996.

2. Kapoor A, Chew P, Silliman RA, Hylek EM, Katz JN, Cabral H and Berlowitz D: Venous thromboembolism after joint replacement in older male veterans with comorbidity. J Am Geriatr Soc 61: 590-601, 2013.

3. Shorr AF and Williams MD: Venous thromboembolism in critically ill patients. Observations from a randomized trial in sepsis. Thromb Haemost 101: 139-144, 2009.

4. Welsby I and Ortel TL: Is it time for individualized thromboprophylaxis regimens in the ICU. Crit Care Med 43: 500-501, 2015.

5. Knudson MM: Venous thromboembolism prophylaxis: One size does not fit all: Comment on 'Comparative effectiveness of unfractionated and low-molecular-weight heparin for prevention of venous thromboembolism following bariatric surgery'. Arch Surg 147: 998-999, 2012.

6. Mahmoudi M and Sobieraj DM: The cost-effectiveness of oral direct factor Xa inhibitors compared with low-molecular-weight heparin for the prevention of venous thromboembolism prophylaxis in total hip or knee replacement surgery. Pharmacotherapy 33: 1333-1340, 2013.

7. Costantino G, Ceriani E, Rusconi AM, Podda GM, Montano N, Duca P, Cattaneo M and Casazza G: Bleeding risk during treatment of acute thrombotic events with subcutaneous LMWH compared to intravenous unfractionated heparin; a systematic review. PLoS One 7: e44553, 2012.

8. Schenkeveld L, Pedersen SS, van Nierop JW, Lenzen MJ, de Jaegere PP, Serruys PW and van Domburg RT: Health-related quality of life and long-term mortality in patients treated with percutaneous coronary intervention. Am Heart J 159: 471-476, 2010.

9. Bergqvist D and Lindblad B: The thromboprophylactic effect of graded elastic compression stockings in combination with dextran 70. Arch Surg 119: 1329-1331, 1984.

10. Jones NA, Webb PJ, Rees RI and Kakkar VV: A physiological study of elastic compression stockings in venous disorders of the leg. Br J Surg 67: 569-572, 1980.

11. Sarmiento A and Goswami AD: Thromboembolic prophylaxis with use of aspirin, exercise and graded elastic stockings or intermittent compression devices in patients managed with total hip arthroplasty. J Bone Joint Surg Am 81: 339-346, 1999.

12. Clagett GP, Anderson FA Jr, Geerts W, Heit JA, Knudson M, Lieberman JR, Merli GJ and Wheeler HB: Prevention of venous thromboembolism. Chest 114 (5 Suppl): 531S-560S, 1998.

13. Wells PS, Anderson DR, Rodger M, Ginsberg JS, Kearon C, Gent M, Turpie AG, Bormanis J, Weitz J, Chamberlain M, et al: Derivation of a simple clinical model to categorize patients probability of pulmonary embolism: Increasing the models utility with the SimpliRED D-dimer. Thromb Haemost 83: 416-420, 2000.

14. Kearon C, Kahn SR, Agnelli G, Goldhaber S, Raskob GE and Comerota AJ; American College of Chest Physicians: Antithrombotic therapy for venous thromboembolic disease: American College of Chest Physicians evidence-based clinical practice guidelines (8th Edition). Chest 133 (6 Suppl): 454S-545S, 2008.

15. van Dongen CJ, Prandoni P, Frulla M, Marchiori A, Prins MH and Hutten BA: Relation between quality of anticoagulant treatment and the development of the postthrombotic syndrome. J Thromb Haemost 3: 939-942, 2005. 
16. Wells PS, Anderson DR, Bormanis J, Guy F, Mitchell M, Gray L, Clement C, Robinson KS and Lewandowski B: Value of assessment of pretest probability of deep-vein thrombosis in clinical management. Lancet 350: 1795-1798, 1997.

17. Heijboer H, Büller HR, Lensing AW, Turpie AG, Colly LP and ten Cate JW: A comparison of real-time compression ultrasonography with impedance plethysmography for the diagnosis of deep-vein thrombosis in symptomatic outpatients. N Engl J Med 329: 1365-1369, 1993.

18. Jaeger KA and Staub D: Ultrasonography for venous thromboembolism. Ultraschall Med 32: 237-240, 2011 (In English and German)

19. Kawaguchi T, Kumabe T, Kanamori M, Nakamura T, Saito R, Yamashita Y, Sonoda Y, Watanabe M and Tominaga T: Early detection of venous thromboembolism in patients with neuroepithelial tumor: Efficacy of screening with serum D-dimer measurements and Doppler ultrasonography. J Neurooncol 101: 495-504, 2011.

20. Chan WS, Spencer FA, Lee AY, Chunilal S, Douketis JD, Rodger M and Ginsberg JS: Safety of withholding anticoagulation in pregnant women with suspected deep vein thrombosis following negative serial compression ultrasound and iliac vein imaging. CMAJ 185: E194-E200, 2013.

21. O'Leary DH, Kane RA and Chase BM: A prospective study of the efficacy of B-scan sonography in the detection of deep venous thrombosis in the lower extremities. J Clin Ultrasound 16: 1-8, 1988.

22. Chiasson TC, Manns BJ and Stelfox HT: An economic evaluation of venous thromboembolism prophylaxis strategies in critically ill trauma patients at risk of bleeding. PLoS Med 6: e1000098, 2009

23. Geerts WH: Prevention of venous thromboembolism in high-risk patients. Hematology AM Soc Hematol Educ Program 2006: 462-466, 2006.

24. De A, Roy P, Garg VK and Pandey NK: Low-molecular-weight heparin and unfractionated heparin in prophylaxis against deep vein thrombosis in critically ill patients undergoing major surgery. Blood Coagul Fibrinolysis 21: 57-61, 2010.

25. Rogers FB, Cipolle MD, Velmahos G, Rozycki G and Luchette FA: Practice management guidelines for the prevention of venous thromboembolism in trauma patients: The EAST practice management guidelines work group. J Trauma 53: 142-164, 2002.

26. Zhao JM, He ML, Xiao ZM, Li TS, Wu H and Jiang H: Different types of intermittent pneumatic compression devices for preventing venous thromboembolism in patients after total hip replacement. Cochrane Database Syst Rev 12: CD009543, 2012.

27. Streiff MB and Lau BD: Thromboprophylaxis in nonsurgical patients. Hematology Am Soc Hematol Educ Program 2012: 631-637, 2012.
28. Larocca A, Cavallo F, Bringhen S, Di Raimondo F, Falanga A, Evangelista A, Cavalli M, Stanevsky A, Corradini P, Pezzatti S, et al: Aspirin or enoxaparin thromboprophylaxis for patients with newly diagnosed multiple myeloma treated with lenalidomide. Blood 119: 933-939, 2012.

29. Jameson SS, Rymaszewska M, Hui AC, James P, Serrano-Pedraza I and Muller SD: Wound complications following rivaroxaban administration: A multicenter comparison with low-molecular-weight heparins for thromboprophylaxis in lower limb arthroplasty. J Bone Joint Surg Am 94: 1554-1558, 2012.

30. Hull RD, Liang $J$ and Townshend G: Long-term low-molecular-weight heparin and the post-thrombotic syndrome: A systematic review. Am J Med 124: 756-765, 2011.

31. Riha GM, Van PY, Differding JA and Schreiber MA; Oregon Health \& Science University Trauma Research Group: Incidence of deep vein thrombosis is increased with $30 \mathrm{mg}$ twice daily dosing of enoxaparin compared with $40 \mathrm{mg}$ daily. Am J Surg 203: 598-602, 2012.

32. Dennis M, Sandercock P, Reid J, Graham C and Forbes J; CLOTS Trials Collaboration: Does intermittent pneumatic compression reduce the risk of post stroke deep vein thrombosis? The CLOTS 3 trial: Study protocol for a randomized controlled trial. Trials 13: 26, 2012.

33. Sekine Y and Koh E: Thoracic surgery for patients with deep vein thrombosis. Kyobu Geka 65: 697-700, 2012 (In Japanese).

34. Comerota AJ, Chouhan V, Harada RN, Sun L, Hosking J, Veermansunemi R, Comerota AJ Jr, Schlappy D and Rao AK: The fibrinolytic effects of intermittent pneumatic compression: Mechanism of enhanced fibrinolysis. Ann Surg 226: 306-313, 1997.

35. Carter C, Kelton J, Hirsh J, Cerskus A, Santos A and Gent M: The relationship between the hemorrhagic and antithrombotic properties of low molecular weight heparin in rabbits. Blood 59: $1239-1245,1982$.

36. Greer IA and Nelson-Piercy C: Low-molecular-weight heparins for thromboprophylaxis and treatment of venous thromboembolism in pregnancy: A systematic review of safety and efficacy. Blood 106: 401-407, 2005.

37. Rota E, Bazzan M and Fantino G: Fondaparinux-related thrombocytopenia in a previous low-molecular-weight heparin (LMWH)-induced heparin-induced thrombocytopenia (HIT). Thromb Haemost 99: 779-781, 2008. 